\title{
Did the Babylonian Astronomers Possess Telescopes?*
}

\author{
Their Observations of Venus, Mars, and Saturn \\ By Dr. Heinrich Hein
}

$\mathrm{T}$ HE two planets which stand closest to the sun, Mercury and Venus, when observed through the telescope exhibit a sickle form like that of the moon and for the same reasons. Only one-half of their spherical surface can be illuminated by the sun, and since their orbits are within that of the earth they must necessarily turn towards the earth a larger or smaller portion of their non-illuminated noctural side. Consequently the portion of the illuminated diurnal side of these planets which we are capable of still seeing from the surface of the earth passes through phases like those of the moon. Fig. 1 shows the apparent magnitude and varying form of Venus.

It was in the year 1610 that Galileo first gazed upon Venus with his newly constructed telescope and perceived her sicklelike form. In none of the writings of the ancient Greeks and Romans and in none of the astronomical works of the Arabs is there any indication that anyone before Galileo had ever perceived the sickle form of Venus. It is not until very recently that any uncertainty has been felt as to whether Galileo was really the first discoverer of the phases of Venus. Some years ago, however, the following prophecy was discovered written in cuneiform letters and uttered by some Babylonian astrologer :

"When it cometh to pass that Venus hideth a star with her right horn and when Venus is large and the star is small, then will the King of Elam be strong and mighty, holding sway over the four corners of the earth, and other kings will pay him tribute."

This is then immediately repeated with the exception that the word right is replaced by left and the name Elam by that of Akkad. Akkad signifies Babylonia, the arch enemy of Elam, and these two nations were in ancient days (cc. 2,000 B.C.) the only two great powers of Asia Minor, and were engaged in a struggle with each other for the mastery of the world as known to them, i.e., for the rulership over the whole of Asia Minor. The blotting out of a small star by the "horn" of Venus must have been such an extraordinary occurrence as to induce the astrologer to connect with it the greatest prize in his power to offer, dominion over the four corners of the world.

There is absolutely no doubt that the Babylonian word in

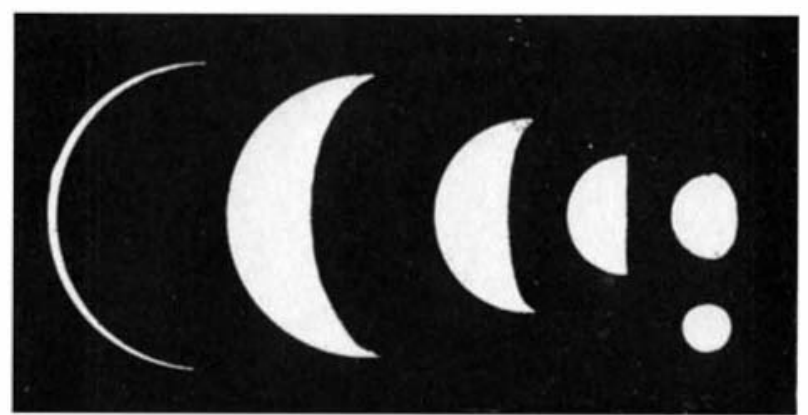

FIG. 1. THE PHASES OF VENUS SHOWING THE CORRECT PROPORTIONAL MAGNITUDES; ABOUT 60": 55" : 38" : $25^{\prime \prime}: 16^{\prime \prime}: 9 "$

question signifies horn. Hence it really looks as if the Babylonians had recognized the phases of Venus. But since they had no telescopes they must obviously have perceived the planet's form resembling a sickle with the naked eye. Yet, as we have said, neither the Greeks nor the Romans were ever able to see the horn of Venus although their astronomers stud-

\footnotetext{
*Translated for the Scientific American Monthly from Kosmos ('Stuttgart), February, 1920.
}

ied the planets zealously enough. In the northern part of Europe the evening star was observed very diligently long before Galileo and yet no one ever perceived its phases.

Hence it has been suggested that the Babylonians must have possessed exceptionally favorable conditions. The air is clearer in that region than in Europe-so clear that bodies upon earth are capable of throwing shadows in the light of Venus-so clear indeed that it is possible to recognize Venus with the naked eye even in the light of day; furthermore, the Babylonians are credited with sharp eyes. A certain Assyriologist assures me indeed that some astronomers have

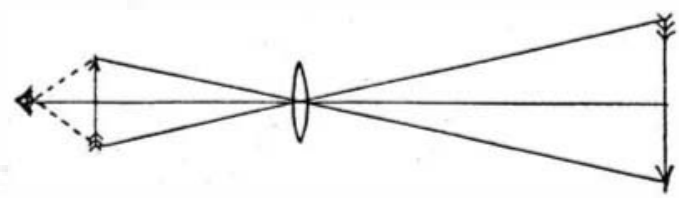

FIG. 2. DIAGRAM OF THE SIMPLE CONVEX PLOYED AS A MAGNIFYING GLASS

been capable of observing the phases of Venus with the naked eye in the clear atmosphere of Oriental lands.

The reasons thus advanced for the thesis in question are not all entirely satisfactory. Even in Europe Venus is capable of throwing shadows under specially favorable circumstances; likewise, we too can see Venus by daylight when conditions are such that the planet possesses her greatest degree of brilliance and when the observer knows exactly that point of the heavens in which to look for her. Anyone who has ever sought to locate the brighter fixed stars at the first fall of night knows how difficult it often is to find them at first and how readily they can be found again after they have been once located. Napoleon I once saw Venus in the full light of day upon an occasion when he chanced to cast his eye upon the right spot in the heavens and thereafter counted the planet as his special star of fortune. As for the supposed keen eyesight of the Babylonians it certainly seems peculiar that the Arabian astronomers who searched the heavens very diligently in later centuries under exactly the same conditions, had no knowledge of Venus's changing form.

As for myself I do not consider it impossible to perceive with the naked eye a certain degree of elongation in an object of the size and form of Venus when she possesses her greatest brilliance and at a time when she appears both somewhat larger and somewhat narrower (for Venus does not possess her greatest apparent magnitude at the time of her greatest brilliance). But in such a case such an object could not be so bright as Venus, for the reason that very bright objects appear to illuminate the retina over a much larger circumference than is actually the case-they appear to be enlarged. The arc of an arc light is no larger than a candle fiame or a gas fiame of the kind formerly used, i.e., without a mantle. From a great distance the arc light appears like a brightly radiant disk, whereas the candle or gas flame at the same distance will appear to be merely a yellow point of light. Similarly it is a well known fact that the brighter fixed stars appear to the human eye larger than those whose light is fainter, in spite of the fact that even in the strongest telescopes none of the fixed stars exhibits a perceptible diameter, but they all resemble mere points. ${ }^{1}$ It is the same with Venus. Human beings are found rarely free from such a super-exciIIt is true also that the brighter fixed stars appear to the human eye perceptibly

none of the fixed stars exhibit a measurable diameter even in the strongest telescopes, and must therefore be recorded as mere points of light. 
tation (irradiation) of the retina. One such individual was the astronomer Heis, who was able to recognize Mercury, Venus, and Jupiter in the bright light of day, and who perceived all the fixed stars as mere points, and who was able to distinguish eleven stars in the Pleiades, while the normal person perceives only six, and some few people can see seven or eight. I have never heard that Heis was able to recognize the phases of Vemus, but if he could not do so this would weigh against the theory that the Babylonians were able to do so, as it must likewise weigh against this theory that the Babylonians recognized only- seven stars in the Pleiades.

Thus it seems to me highly improbable that the Babylonians were capable of observing the phases of Venus with the naked eye, if not entirely impossible. I have, therefore, pondered the question as to whether the Babylonians did not perhaps possess some sort of magnifying instruments. We know that the ancients were acquainted with hollow mirrors, which were used as "shaving mirrors" are among us, to magnify the face and which were also used in Greece, for example, like burning glasses in order to kindle the sacred sacrificial fire by the light of the sun itself. We are told, too, that somewhat later Archimedes kept the Roman fleet away from the harbor of beleaguered Syracuse by means of powerful "burning glasses." It occurred to me, therefore, that the observation of the phases of Venus must have been made possible to the Babylonians by means of the burning glass or hollow

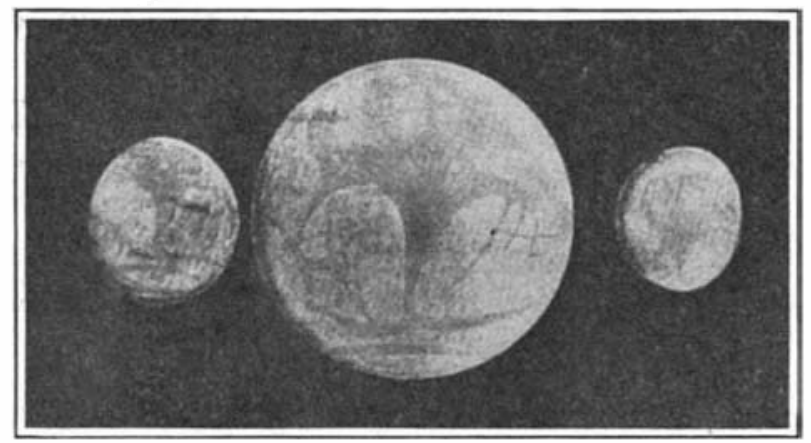

FIG. 3. MARS PHASES

PHAS'ES 11": 9"

mirror. Everyone understands the method by which a burning glass consisting of a convex lens operates. Every one at one time or another has thrown an image of the sun upon his hand with such a glass or burnt a hole in a sheet of paper with it. The distance of the image of the sun from the lens is called the "focal distance" of the lens. At this distance not only an image of the sun is formed but likewise images of all objects which are located at very great distances from the lens. This is readily verified by using a lens at night to experiment with distant sources of light, such as bright windows, lanterns, etc. When the luminous object comes considerably closer to the lens its image recedes from the lens; however, this fact does not here enter into the question.

Let us assume for example that the lens has a focal distance of one meter-this signifies that the lens will form an image of the sun at a distance of one meter from itself. But it is likewise true that an object only 100 meters distant will throw an image at the same distance of one meter (practically speaking), but we must remember that the respective magnitudes of the object an the image bear the same relation to each other as do their distances, i.e., in the instances referred to the object will be 100 times as large as the image. Again the diameter of the sun might be calculated with the same laws. The sun is 150 millions $\mathrm{km}$. distant from the earth. Hence if the sun's image is formed at a distance of one meter this implies that the sun is $\mathbf{1 5 0}$ billion times as far from the lens of the image. Thus we need only measure the diameter of the sun's image in millimeters and multiply it by 150 billions and we shall obtain the diameter of the sun in millimeters.
Suppose that the object situated at a distance of 100 meters is a brightly lighted window 100 centimeters high and observed in the evening. The image will be situated at a distance of 1 meter and will be exactly 1 centimeter high. It can be received upon a pane of "ground glass. If we now observe the lighted window from a distance of 100 meters and the image from a distance of 1 meter, it is obvious that both

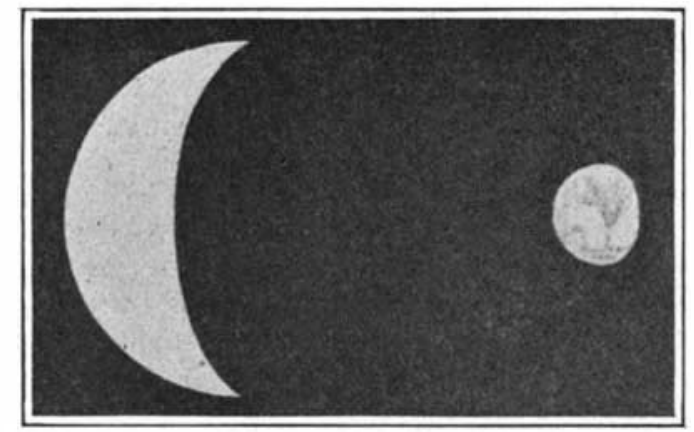

FIG. 4. THE MOST CLEARLY MARKED PHASES OF VENUS AND OF MARS SHOWN IN THETR CORRECT PROPORTION WITH RESIPECT TO MAGNITUDE AND TO BRIGHTNES:S : 55" AND 13"

will appear of the same size to the eye. (Thus from the view point of the lens the object in the image always appears the same size.) If we now desire to see the window appear four times as large we must approach the actual window until we are only 25 meters distant. However, this fourfold enlargement can be obtained more conveniently by observing the image at a distance of 25 centimeters. Then the image will appear four times as great as the object at 100 meters' distance. If the image has been received upon a screen of ground glass, then it can be observed from either side and the eye can approach the image until the required degree of magnification is obtained. If the image be regarded from the rear side of the ground glass screen-in which case the screen stands between the lens and the eye-the screen may be slowly moved forward. We then observe that the image slowly moves down the screen until it finally hovers freely in space at the

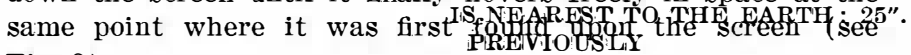
Fig. 2).

The matter may be summed up, therefore, as follows: The object at a distance of 100 meters appears to be of the same size as the image at a distance of 1 meter. Since, however, the eye, is not forced to regard the image at the distance of exactly 1 meter, but can without trouble observe it at a distance of $25 \mathrm{~cm}$. it is possible to obtain with a simple lens

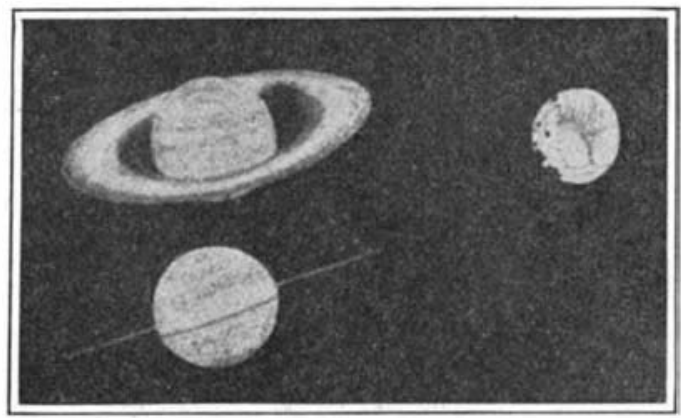

FIG. 5. THE MOST MARKED PHASES OF SATURN AND OF MARS SHOWN IN THEIR CORREC'T PROPORTIONS AS TO MAGNITUDE. THE BRIGHTNESS OF MARS EXCEEDS THAT OF SATURN

THAT OF MARS (FIG. 4). 16" TIMES 42" AND 13"

having a focal distance of 1 meter a fourfold enlargement of objects at $x$ great distance. It is my belief that this use of the simple convex lens as a telescope is entirely new, although such lenses have been employed as microscopes for centuries.

Anyone who has a spectacle lens of very slightly convex form can easily construct a one lens telescope for himself. The only thing required is a cardboard tube. The focal dis- 
tance of the lens is first determined, which is readily done by using it to form an image of the sun or the moon and then measuring the distance from the lens to the spot at which the said image is most sharply defined. Suppose that the focal distance happens to be 75 centimeters. Twenty-five centimeters is then added to this and the tube is made $100 \mathrm{~cm}$. long. The lens is inserted at the forward end of the tube and the eye is place at the rear end in order to observe any given object located at a great distance. It will be found that the said object appears as an inverted magnified image. The field of vision is exactly as large as te lens and is, therefore, of no significance. When an ordinary spectacle lens is employe the image is somewhat distorted. The placing of the eye presents the chief difficulty. The best method is to place a diaphragm in the tube at a distance of about $25 \mathrm{~cm}$. from the eye at about the point where the image would be formed. The eye then has an indication as to where it can be probably best placed. Anyone who has a long telescope tube needs only remove the rear lens, but in this case the eye must generally be place 15 to $20 \mathrm{~cm}$. to the rear since telescope tubes can rarely be extended to so great a length as is here necessary. It is now obvious that with a lens having a focal distance of 10 meters it is possible to obtain a magnification of 40 diameters. The same thing holds true with |regard to concave mirrors as to the double convex lens employed above, with the exception

mirror instead of through it.

It was not until a considerable time after I had come to the conclusion recorded above that I learned that certain Babylonian texts also speak of the horns of Mars. Since the Babylonians were also accustomed to apply the term "horn" to very slight swellings, it is possible that they had reference to the phases of Mars, though these are small (See Fig. 3).

Under the best circumstances we are able to see only about one-sixth of the dark nocturnal side of Mars. But Mars is so very far distant from the earth that it is probably impossible for the naked eye to perceive the disk distorted. Its diameter is hardly 15", whereas Venus exhibits a diameter of 55" at the period when the curved sickle form is plainest to be seen (see Fig. 4). Hence it would be probably impossible for the unassisted eye to perceive a slight elongation of the planet. Thus the Babylonian reference to the horns of Mars likewise raises the question as to whether the Babylonians may not have possessed primitive instruments capable of telescopic observations-a question which I believe can be answere in the affirmative, at least as regards the possibility.

However, in spite of this possibility I do not believe that the Babylonians really did make use of the concave mirror as a telescope. Such mirrors must be ground in the form of a portion of a whole sphere, or, better still, in parabolic form. Such grinding requires machines, whereas it is certain that the mirrors ground by hand which the Babylonians possessed were of short focal distance and such mirrors produce distorted images. Then, too, if they were able to recognize the phases of Mars they certainly must also have known something about the form of Saturn. The diameter of the latter is about 16", and the largest diameter of the ring is about $40^{\prime \prime}$. As we know, the ring disappears at times when we are looking straight at its edge. This phenomenon, that Saturn possesses "horns" which it loses at times should not have escaped the observation of the Babylonians and must have intrigued their imagination. In case records of such observations are discovered with respect to Saturn they would certainly furnish testimony in favor of the Babylonian's use of the coneave mirror as a telescope. ${ }^{2}$ Until such a text is discotedent mbserver must ever, I shall be of opinion that the mention of the "horns" of Venus and of Mars refer to the phenomena of refraction in the air by means of which the former planet is distorted to resemble a narrow line, which might possibly extend to a small fixed star. Such an occurrence would be an exceedingly rare phenomenon, justifying an extraordinary prophecy.

${ }^{2}$ The late M. W. Meyer mentions somewhere the representation of the planet Saturn with rings by the Babylonians. However, the Babylonians sometimes represent other planets also as surrounded by rings. In my opinion only a direct mention of the rings in the cuneiform text would be convincing. And we must recall, too, how difficult the astronomers of the 17th century found it to recognize the true nature of the rings in spite of the possession of comparatively good instruments.

\title{
The Origin of Comets*
}

\section{Do They Form a Part of Our Solar System?}

\author{
By Elis Strömgren \\ Director of the Observatory of Copenhagen
}

The following article is an abstract made by the author himself, the distinguished Danish astronomer, Prof. STRöMGREN, of a longer article published in Scientia (Bologna) for August, 1918.-EDITOR.

A LL comets known to us have been observed within our own solar system. Our sun is one of billions of suns to whose ensemble the name is given of the Galactic System, and in all probability there are million of systems similar to our own galactic system.

Our little solar system is composed of the sun, of the planets with their satellites, and of comets. It is with the latter that the present article deals and we shall confine ourselves to the study of the following questions:

Whonce do comets come and whither do they go? Do they form a part of our own solar system, or are they, as it were, migratory birds coming from afar and folding their wings for but a moment upon the edge of our own little nest in the heavens?

Ever since man has been capable of meditating upon those things which lie beyond his immediate grasp, these questions

*Translated for the Scientific American Monthly from L'Astronomie (Paris), for October, 1919. have exerted a profound attraction upon his imagination. The opinions of astronomers upon this point have long been divided and changing, but today we are in a condition to give a positive answer to the question as to the origin of comets.

The law of gravitation is the basis of all calculations relating to the orbits of the celestial bodies, and if this law were unknown to us it would be impossible to conceive of a discussion of the problem of celestial movements. We are all familiar with the method by which the effect of this force upon two celestial bodies - the sun and the earth, for example-can be demonstrated. The small body, $i$. $e$., the earth, is forced to describe an elliptical orbit about the larger body, $i$. $e$. the sun. Such is the orbit of the earth and such also are the orbits of the other planets.

However, the mathematical study of the problem shows, as every one knows, that the problem of two bodies comprises the possibility of two other kinds of orbits, namely the parabola and the hyperbola. As we know, the ellipse consists of a closed curve while the parabola and the hyperbola are open curves whose branches are of infinite extent.

As a result of this an elliptical orbit in the case of a comet indicates that the comet belongs to our own solar system, while an orbit, which is either paraboilc or hyperbolic indicates that 